\title{
Primary localized amyloidoma of the renal pelvis: A case report and literature review
}

\author{
WEI LU ${ }^{1-3^{*}}$, YANJUN WANG ${ }^{2 *}$, MENG ZHANG ${ }^{1-3^{*}}$, YONGHONG $\mathrm{LI}^{2}, \mathrm{YUN} \mathrm{CAO}^{4}$, \\ YONGBO XIAO ${ }^{4}$, ZHIMING CAI $^{1,3}$, SONG WU $^{2,3}$ and FANGJIAN ZHOU ${ }^{2}$ \\ ${ }^{1}$ Department of Graduate School, Anhui Medical University, Hefei, Anhui 230022; \\ ${ }^{2}$ Department of Urology, State Key Laboratory of Oncology in Southern China, Sun Yat-sen University Cancer Center, \\ Guangzhou, Guangdong 510060; ${ }^{3}$ Department of Urology, Shenzhen Second People's Hospital, \\ The First Affiliated Hospital of Shenzhen University, Shenzhen, Guangdong 518035; ${ }^{4}$ Department of Pathology, \\ State Key Laboratory of Oncology in Southern China, Sun Yat-sen University Cancer Center, \\ Guangzhou, Guangdong 510060, P.R. China
}

Received February 5, 2015; Accepted November 19, 2015

DOI: $10.3892 / \mathrm{ol} .2015 .4038$

\begin{abstract}
Primary localized amyloidomas of the renal pelvis are challenging to diagnose, due to non-specific imaging results and the unusual location. The present study reports a rare case of primary localized amyloidoma of the renal pelvis and aims to illustrate the challenges in pre-operatively discriminating between this disease and transitional cell carcinomas. The present study identified that the mass was situated in the left renal pelvis using ultrasonography. A nephroureterectomy was performed following careful preparation. Finally, histopathological studies revealed that the tumor consisted of massive diffuse deposits of amyloid and microscopic amorphous eosinophilic material, which stained positively for Congo red, demonstrating potassium permanganate digestion. Consequently, a diagnosis of amyloid light chain-type amyloidoma was determined. Systematic examinations were performed following the unexpected diagnosis, which eliminated the possibility of amyloid associated-type amyloidoma. In total, 4 months post-surgery, the patient remained tumor-free.
\end{abstract}

Correspondence to: Professor Fangjian Zhou, Department of Urology, State Key Laboratory of Oncology in Southern China, Sun Yat-sen University Cancer Center, 651 Dong Feng Road East, Guangzhou, Guangdong 510060, P.R. China

Email: zhoufj@sysucc.org.cn

Professor Zhiming Cai, Department of Graduate School, Anhui Medical University, 81 Meishan Road, Shushan District, Hefei, Anhui 230022, P.R. China

E-mail: caizhiming2000@hotmail.com

${ }^{*}$ Contributed equally

Key words: amyloidoma, renal pelvis, nephrectomy

\section{Introduction}

Localized amyloidoma is generally divided into two styles: AL-type and AA-type. AL-type occurs with an immunocyte dyscrasia while AA-type occurs with chronic infection, non-immunocyte neoplasia or inflammation. Localized amyloidoma occurs most often in the mediastinum or abdomen. Although amyloidomas may occur in almost all organ systems in the body; however, primary amyloidoma of the renal pelvis is rare. Using the keywords: 'renal pelvis', 'amyloidoma' and 'amyloid tumor' and search terms (renal pelvis) and (amyloidoma or amyloid tumor) in PubMed (National Center for Biotechnology Information, U.S. National Library of Medicine, Bethesda, MD, USA), a literature search was performed and only 26 cases of primary amyloidomas of the renal pelvis were identified (Table I) (1-23). Primary localized amyloidoma may present as hematuria and lumbago (14). A primary localized amyloidoma of the renal pelvis consists of amyloid deposits that may present as malignant tumors (15). The present study reports the rare case of a patient with amyloidoma that was confined to the renal pelvis, and the patient exhibited similar symptoms to those of upper urinary tract transitional cell carcinoma (TCC). In addition, 26 cases of patients with primary amyloidoma of the renal pelvis identified from the literature were also reviewed. Nephrectomy was the most selected treatment in the reported cases. The majority of patients achieved long time disease free survival and good prognosis. (7). The clinical and pathological features of the cases were discussed, in particular, the treatment methods and prognosis.

\section{Case report}

A 56-year-old man presented to the Department of Urology, Sun-Yat Sen University Cancer Center (Guangzhou, China) with left flank pain, symptoms of urinary irritation and intermittent gross hematuria. The patient had experienced the symptoms for 6 years; however, in the month prior to presentation, the 


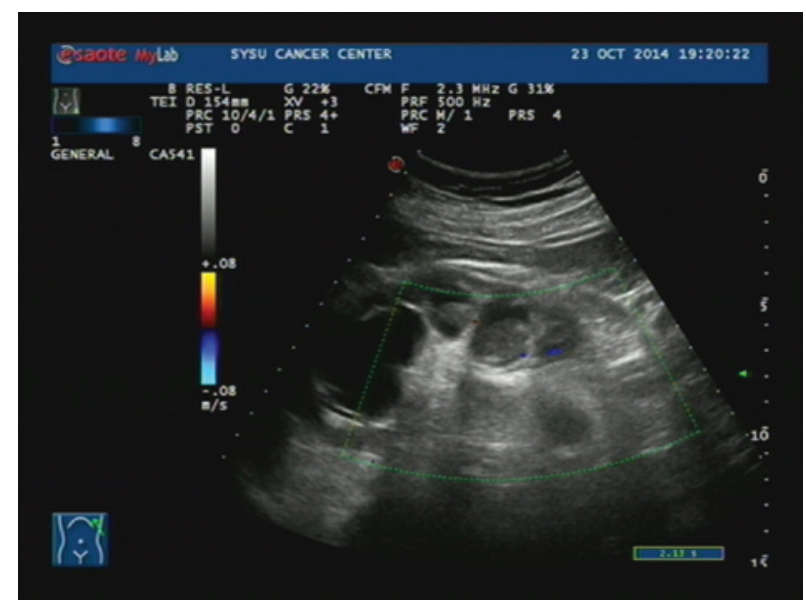

Figure 1. Ultrasound scans revealed that the mass exhibited a low heterogeneous echo with an irregular shape, and was located in the left renal pelvis and measured $55 \times 19 \mathrm{~mm}$ in size.
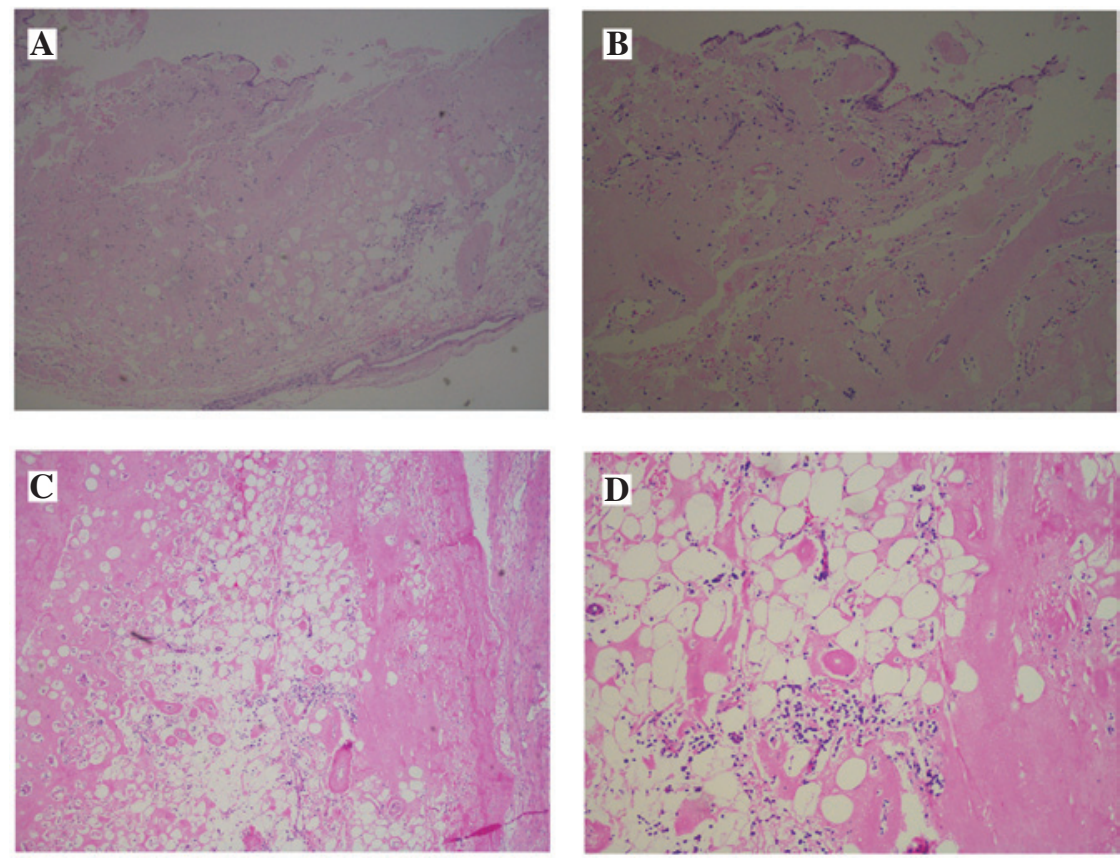

Figure 2. Light microscopy revealed that amyloid had been deposited in the renal pelvis, fat and vasculature. Massive amyloid deposits were located in the renal pelvis [magnification, (A) x40 and (B) x100] and the vasculature [magnification, (C) x40 and (D) x100].
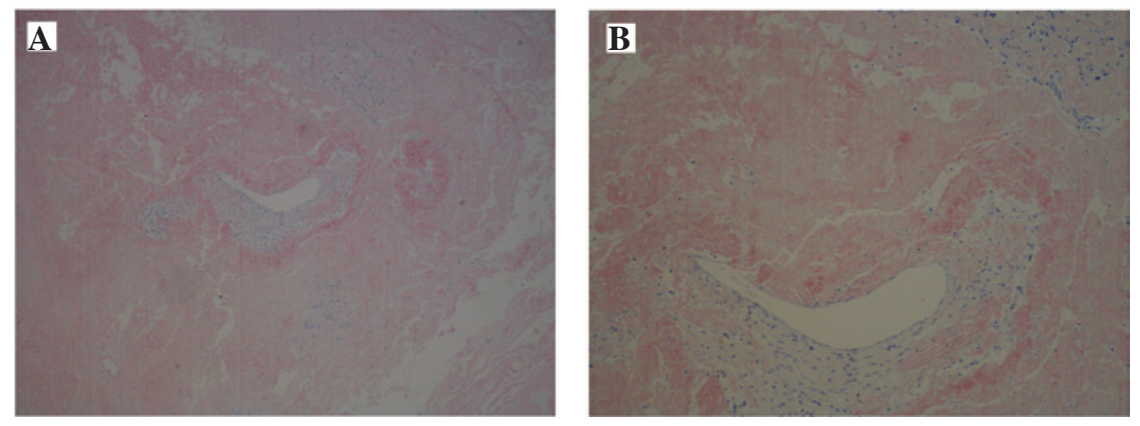

Figure 3. Congo red stain revealed the presence of a red-brown material in the renal pelvis and vasculature. Magnification, (A) x40 and (B) x100.

symptoms had become worse. Ultrasonography revealed a mass with a low-intensity heterogeneous pattern that measured $55 \times 19 \mathrm{~mm}$ in size and obstructed the lumen of the renal pelvis. Dilatation of the renal pelvis was located in the left kidney (Fig. 1). A preliminary diagnosis of TCC of the renal pelvis was suggested based on sonography, and therefore, a 


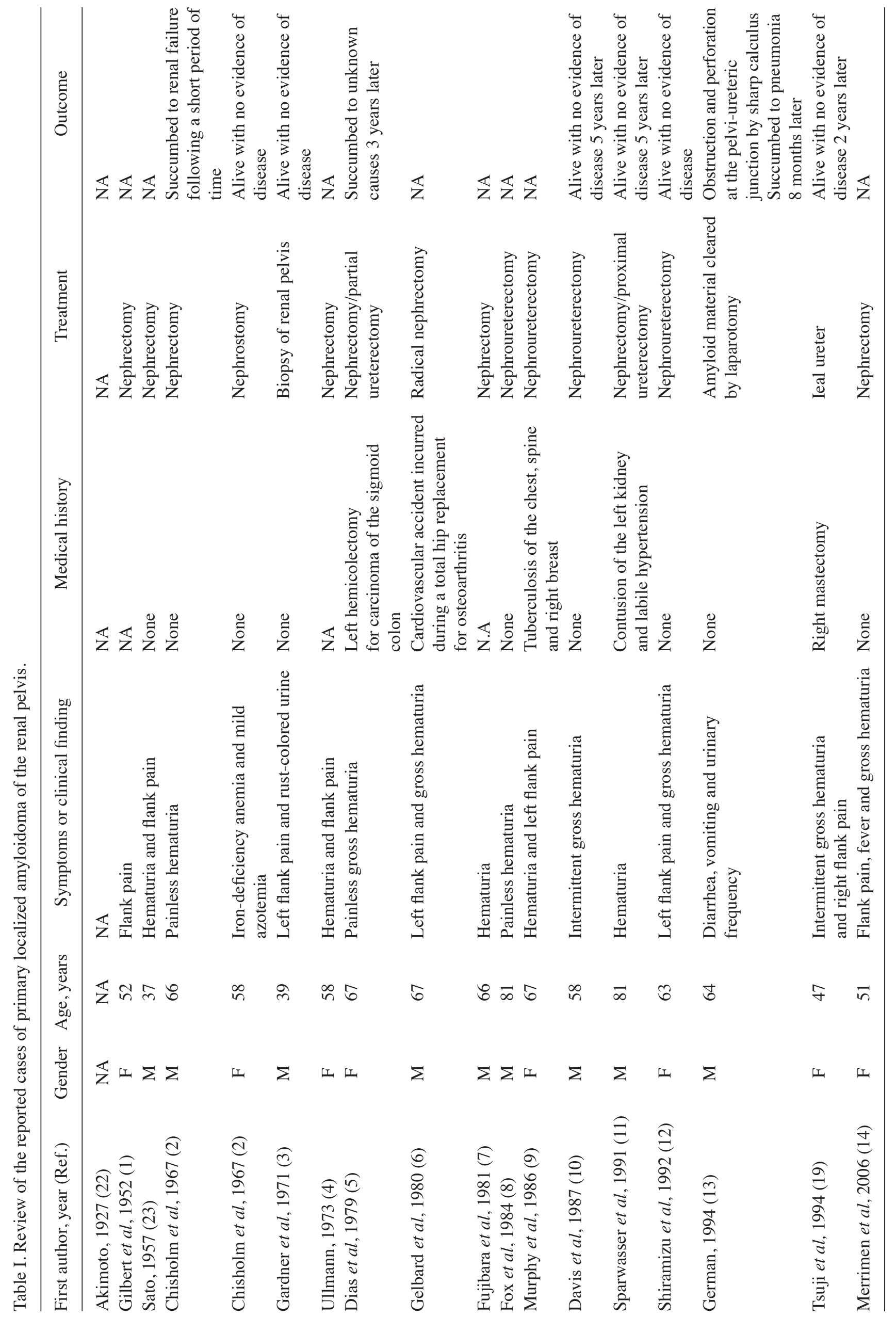




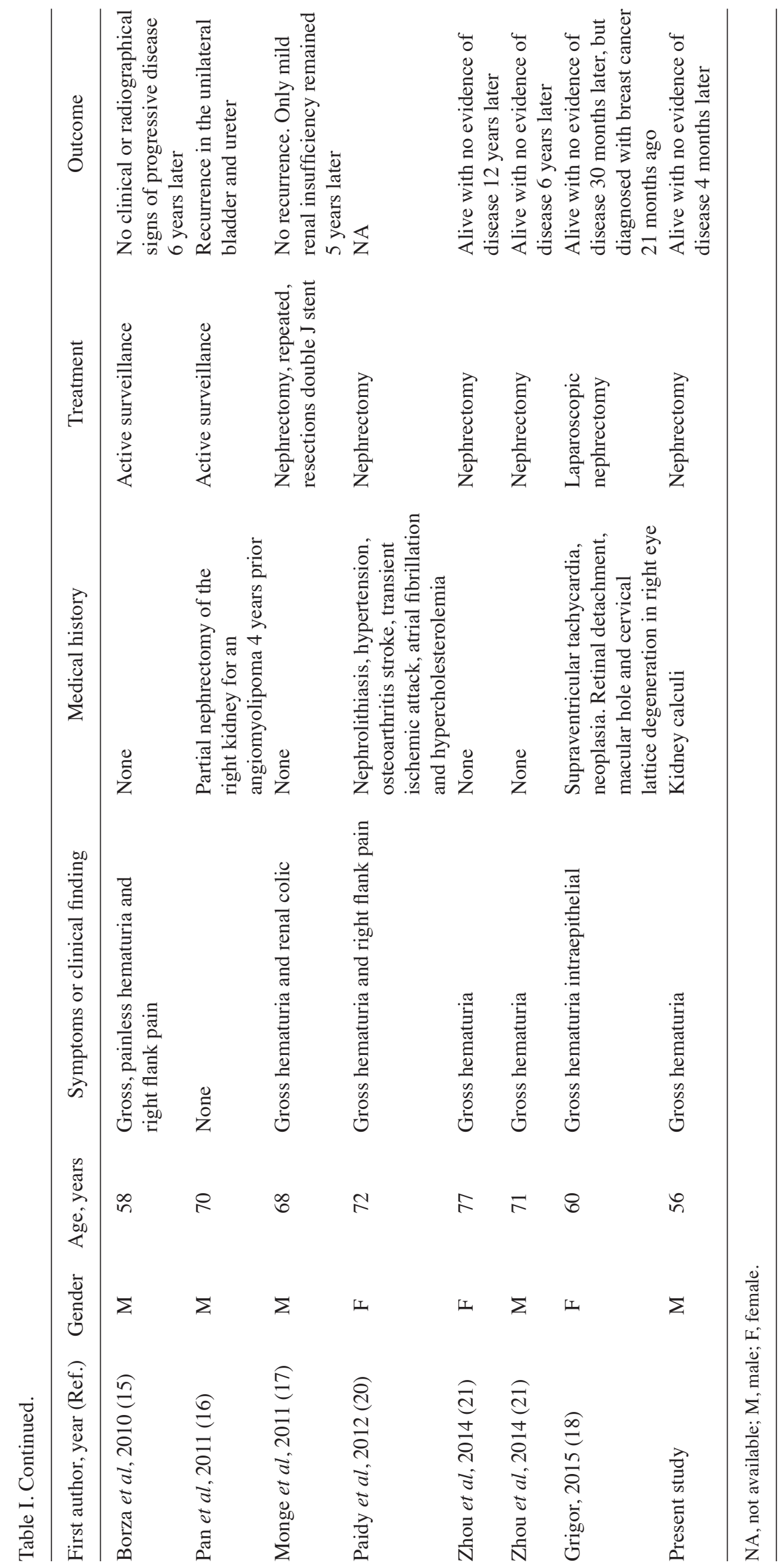


nephroureterectomy was advised. However, 5 urinary cytology tests were negative and pre-operative examinations revealed no abnormal signs, such as abdominal tenderness or rebound pain, during physical examination. Blood tests yielded the following results: Urine protein, 2+; urine red blood cell count, $>3$ cells/high power field; urine erythrocytes, $932 \mu \mathrm{l} / \mathrm{l}$; left renal glomerular fitration rate, $18 \mathrm{ml} / \mathrm{min}$; right renal glomerular fitration rate, $64 \mathrm{ml} / \mathrm{min}$; and the serum creatinine levels, eosinophil numbers and basophil numbers were within the normal ranges. The patient had been a smoker for 20 years and had suffered from nephrolithiasis for 6 years. The patient possessed no known drug allergies. Subsequently, the patient agreed to undergo a nephroureterectomy.

Following surgery, the excised mass underwent additional tests. Macroscopically, the surgical specimen revealed tumors located in the renal pelvis. No clear difference in the ureter was observed. A cut section of the tumor revealed a red-yellow surface with firm regions. Microscopically, histopathological studies revealed that the tumor consisted of massive diffuse deposits of amyloid and microscopic, eosinophilic, amorphous material (Fig. 2) and an absence of neoplastic cells. The tumor stained positive for Congo red (Fig. 3), which indicates the presence of material that is retained following potassium permanganate digestion. A final diagnosis of amyloid light chain-type amyloidoma of the renal pelvis was determined. The patient received regular surveillance and was alive with no evidence of disease 5 months later.

Written informed consent was obtained from the patient for the publication of the present study.

\section{Discussion}

Amyloidosis refers to a large heterogeneous group of diseases that is characterized by extracellular deposits of amyloid in individual organs or tissue. Amyloid is an amorphous, insoluble and proteinaceous material (17). Extracellular amyloidosis consists of specific protein fibrils (24). Amyloidosis can be classified into 4 groups, consisting of primary, secondary, heredofamilial and $\beta 2$-microglobulin-associated amyloidosis. This disease may be additionally classified as localized amyloidosis, which involves a single organ, or systemic amyloidosis, which involves multiple organs and is the most common classification among reported cases (6). By contrast, localized amyloidosis occurs much less frequently. (15) Systemic amyloidosis may be primary, progressive and fatal. Primary amyloidosis is commonly associated with an underlying immune dyscrasia, such as multiple myeloma and Waldenstrom's macroglobulinemia (15).

The etiology of primary localized urinary amyloidosis remains unknown; however, it may be possible that amyloid deposits are produced locally, or the submucosa of the genitourinary tract may be targeted by light-chains that are produced elsewhere. Numerous studies support the first hypothesis, as there is an absence of a monoclonal plasma component. An additional hypothesis is that protein deposits in bladder non-amyloid associated (AA) localized amyloidosis consist of the immunoglobulin $\lambda$ light-chain subgroup I or IV (17).

Furthermore, non-AA localized amyloidosis has been described in the lung (25), nervous system (26), skin (24), larynx (27), intestinal tract (28) and genitourinary tract (17).
Using PubMed, a literature search was performed and only 26 cases of primary amyloidomas of the renal pelvis were identified. The inclusion and exclusion criteria is whether primary localized amyloidoma. The majority of reported cases concerned with localized urinary tract amyloidosis are characterized as primary type, but secondary localized amyloidosis has been reported without a chronic systemic inflammatory state. Renal pelvis primary localized amyloidoma is an extremely rare condition, and is notable due to its clinical presentation and radiographic appearance, which mimics that of transitional cell carcinoma. In a review of the English and French literature by Monge et al (17), 169 cases of genitourinary tract localized amyloidoma were reported over the past 100 years. The renal pelvis was the most rare location identified, accounting for $\sim 6 \%$ of the 169 cases, which was lower than the number located in the bladder, ureter and urethra. To the best of our knowledge, only 26 cases of renal pelvis primary localized amyloidoma have been reported (1-23).

Positive staining for Congo red is used to diagnose primary localized amyloidoma. In order to exclude AA-type amyloidosis, screening by pre-exposure of tissue slides to $\mathrm{KMnO}_{4}$ stain is performed, since false-negative results may be obtained using immunohistochemistry (negative for Congo red) (17). Clinical symptoms of primary localized amyloidoma of the renal pelvis typically present as gross hematuria, flank pain and urinary irritative symptoms, which mimic the symptoms of inflammation and neoplasm (21). It is challenging to distinguish between amyloidoma and upper urinary tract TCC solely from radiological findings, which may be non-specific (17). In addition, urine cytology does little to contribute to the diagnosis, since the majority of amyloid deposits are subepithelial. Consequently, to avoid misdiagnosis, upper urinary tract tumors should be evaluated microscopically by ureteroscopic biopsy when multiple urine cytology analyses are negative, or if possible, by surgical biopsy using frozen tissue sections examined prior to radical resection.

Primary localized amyloidoma possesses a relatively good prognosis in the genitourinary tract and other organ systems. Recurrence and implantation of the tumor has not been identified in reported cases. Nephrectomy was the chosen treatment in the present study, and is used in the majority of reported cases. However, by monitoring the progression of the primary localized amyloid tumor in the renal pelvis, using active surveillance with serial imageological examination, a similar outcome to nephrectomy may be observed (15). This should be considered by all urologists.

Primary localized amyloidoma is extremely rare in the renal pelvis $(14,15)$. Consequently, there is a lack of standardized clinical symptoms and specific laboratory tests, and in addition, imageological examination mimics TCC. A pre-operative ureteroscopic biopsy or surgical biopsy is required when multiple urinary cytology analyses are not positive or a benign tumor, including primary localized amyloidoma, is suspected, to avoid an unnecessary nephroureterectomy. Early radical surgery is not required unless there is no renal function or a severe urinary tract obstruction. Instead, active surveillance with serial imageological examination may be used to monitor the progression of the lesion. In conclusion, primary localized amyloidoma of renal pelvis is a benign and rare tumor, which has a relatively good prognosis. 


\section{References}

1. Gilbert LW and McDonald JR: Primary amyloidosis of the renal pelvis and ureter: Report of case. J Urol 68: 137-139, 1952.

2. Chisholm GD, Cooter NB and Dawson JM: Primary amyloidosis of the renal pelvis. BMJ 1: 736-738, 1967.

3. Gardner KD Jr, Castellino RA, Kempson R, Young BW and Stamey TA: Primary amyloidosis of the renal pelvis. N Engl J Med 284: 1196-1198, 1971.

4. Ullmann AS: Primary amyloidosis of the renal pelvis: A case report and review of literature. Mich Med 72: 29-33, 1973.

5. Dias R, Fernandes M, Patel RC, de Shadarevian JJ and Lavengood RW: Amyloidosis of renal pelvis and urinary bladder. Urology 14: 401-404, 1979.

6. Gelbard M and Johnson S: Primary amyloidosis of renal pelvis and renal cortical adenoma. Urology 15: 614-617, 1980.

7. Fujihara S and Glenner GG: Primary localized amyloidosis of the genitourinary tract: Immunohistochemical study on eleven cases. Lab Invest 44: 55-60, 1981.

8. Fox M, Hammond JC, Knox R and Underwood JC: Localised primary amyloidosis of the renal pelvis. Br J Urol 56: 223-224, 1984.

9. Murphy MN, Alguacil-Garcia A and MacDonald RG: Primary amyloidosis of renal pelvis with duplicate collecting system. Urology 27: 470-473, 1986.

10. Davis PS, Babaria A, March DE and Goldberg RD: Primary amyloidosis of the ureter and renal pelvis. Urol Radiol 9: 158-160, 1987.

11. Sparwasser C, Gilbert P, Mohr W and Linke RP: Unilateral extended amyloidosis of the renal pelvis and ureter: A case report. Urol Int 46: 208-210, 1991.

12. Shiramizu M, Nakamura K, Baba S, Katsuoka $Y$ and Kinoshita H: Primary localized amyloidosis of the renal pelvis coexisting with transitional cell carcinoma: A case report. Hinyokika Kiyo 38: 699-702, 1992.

13. German KA and Morgan RJ: Primary amyloidosis of the renal pelvis and upper ureter. Br J Urol 73: 99-100, 1994.

14. Merrimen JL, Alkhudair WK and Gupta R: Localized amyloidosis of the urinary tract: Case series of nine patients. Urology 67: 904-909, 2006.

15. Borza T, Shah RB, Faerber GJ and Wolf JS, Jr: Localized amyloidosis of the upper urinary tract: A case series of three patients managed with reconstructive surgery or surveillance. J Endourol 24: 641-644, 2010.

16. Pan DL and Na YQ: Amyloidosis of the unilateral renal pelvis, ureter and urinary bladder: A case report. Chin Med Sci J 26: 197-200, 2011.
17. Monge M, Chauveau D, Cordonnier C, Noël LH, Presne C, Makdassi R, Jauréguy M, Lecaque C, Renou M, Grünfeld JP, et al: Localized amyloidosis of the genitourinary tract: Report of 5 new cases and review of the literature. Medicine (Baltimore) 90: 212-222, 2011.

18. Grigor T and Munro N: Amyloidosis of the renal pelvis: A harbinger of mammary carcinoma? BMJ Case Rep 16: 2015, 2015.

19. Tsuji Y, Michinaga S and Ariyoshi A: Ileal ureter: Another option for the treatment of localized amyloidosis of the upper urinary tract. J Urol 151: 999-1000, 1994.

20. Paidy S, Unold D and Catanzano TM: AIRP best cases in radiologic-pathologic correlation: localized amyloidosis of the renal pelvis. Radiographics : a review publication of the Radiological Society of North America. Inc 32: 2025-2030, 2012.

21. Zhou F, Lee P, Zhou M, Melamed J and Deng FM: Primary localized amyloidosis of the urinary tract frequently mimics neoplasia: A clinicopathologic analysis of 11 cases. Am J Clin Exp Urol 2: 71-75, 2014

22. Akimoto K: Ober amyloidartige Eiweissniederschlage im Nierenbecken. Beitr Pathol Anat 78: 239, 1927.

23. Sato S: Primary amyloidosis of the renal pelvis and ureter: Report of a case. Acta Med Biol 5: 15, 1957.

24. Reitboeck JG, Feldmann R, Loader D, Breier F and Steiner A: Primary cutaneous amyloidoma: A case report. Case Rep Dermatol 6: 264-267, 2014.

25. Dong MJ, Zhao K, Liu ZF, Wang GL and Yang J: Primary pulmonary amyloidosis misdiagnosed as malignancy on dual-time-point fluoro-deoxyglucose positron emission tomography/computed tomography: A case report and review of the literature. Oncol Lett 9: 591-594, 2015.

26. Apostolova LG, Hwang KS, Avila D, Elashoff D, Kohannim O, Teng E, Sokolow S, Jack CR, Jagust WJ, Shaw L, et al; Alzheimer's Disease Neuroimaging Initiative: Brain amyloidosis ascertainment from cognitive, imaging, and peripheral blood protein measures. Neurology 84: 729-737, 2015.

27. Cheng KJ, Wang SQ and Lin S: Localized amyloidosis concurrently involving the nasopharynx, larynx and nasal cavities: A case report. Zhonghua Er Bi Yan Hou Tou Jing Wai Ke Za Zhi 44: 875-876, 2009 (In Chinese).

28. Yaita H, Nakamura S, Kurahara K, Nagasue T, Kochi S, Oshiro Y, Ohshima K, Ikeda Y and Fuchigami T: Primary small-bowel adult T-cell leukemia/lymphoma with gastric AL amyloidosis. Endoscopy 46: E613-614, 2014. 